\title{
Long simulations of the Solar System: Brouwer's Law and chaos
}

\author{
K. R. Grazier* W. I. Newman ${ }^{\dagger} \quad$ James M. Hyman \\ Philip. W. Sharp ${ }^{\S}$
}

(received 28 October 2004, revised 18 October 2005)

\begin{abstract}
The accuracy of long simulations of the Solar System is limited by the accumulation of round-off error. If the round-off error is systematic, the error in conserved quantities grows as $t$ where $t$ is time, and that in dynamical variables as $t^{2}$. If the round-off error is stochastic, the error grows as $t^{1 / 2}$ and $t^{3 / 2}$ respectively.
\end{abstract}

${ }^{*}$ The Jet Propulsion Laboratory, California Institute of Technology, Pasadena, CA 91109, USA. mailto:krg@anlashok.jpl.nasa.gov

$\dagger$ Departments of Earth and Space Sciences, Physics and Astronomy, and Mathematics, University of California, Los Angeles, CA 90095, USA. mailto:win@ucla.edu

†Theoretical Division, Los Alamos National Laboratory, Los Alamos, NM 87545, USA. mailto: hyman@lanl.gov

$\S$ Department of Mathematics, University of Auckland, Private Bag 92019, Auckland, NEW ZEALAND. mailto: sharp@math.auckland.ac.nz

See http://anziamj.austms.org.au/V46/CTAC2004/Gra2 for this article, (c) Austral. Mathematical Soc. 2005. Published October 19, 2005. ISSN 1446-8735 
In a previous study, we showed that it was possible to implement the order thirteen Störmer method so the errors grew stochastically for the two-dimensional Kepler problem. Here we show the implementation gives stochastic error growth on three-dimensional simulations of the Solar System. Our integrations are such that the positions of the major planets are known with an estimated error of no more than $2^{\circ}$ after $10^{9}$ years, a precision unmatched by earlier investigations.

Further, our numerical results suggest the outer Solar System is not chaotic as has previously been reported, but rather computational errors in positions grow no faster than $t^{3 / 2}$, conforming with existing models for stochastic error growth in an otherwise well-behaved system of ordinary differential equations.

\section{Contents}

1 Introduction

C1087

2 Test 1

C1089

3 Test 2

C1093

4 Test 3

C1097

5 Discussion

C1097

References

C1101

\section{Introduction}

As the Sun and planets formed, planetesimals, small solid bodies rich in metals, silicates and possibly ice, underwent a violent dynamical birth, of- 
ten resulting in their amalgamation or accretion into the planets, or being gravitationally ejected from the Solar System. Planetesimals that survived this sorting process provide essential clues to the Solar System's origin and evolution.

These planetesimals reside near separatrices that distinguish them from objects that ultimately fell into the Sun and planets, or were catapulted away. Hence the trajectories of these planetesimals can reside on the edge of chaos and their simulation over long intervals such as one-billion years $[5,6,7,4,11$, e.g.] requires accurate integrations.

Brouwer [2] proved for a fixed step size integration of a non-chaotic system that random round-off error for conserved quantities and dynamical variables will grow as $t^{1 / 2}$ and $t^{3 / 2}$ respectively, where $t$ is time. Brouwer's result thus gives lower bounds on the error growth.

In [8] we presented an implementation of the order thirteen Störmer method ([9, p.462,e.g. $]$ or [10, p.291]) that achieved these bounds on the two-dimensional Kepler problem. The method uses a backward difference, summed form implementation and a technique we call significance-ordered computation. We tested our method on the two-dimensional Kepler problem by performing integrations of ten million orbit for eccentricities of 0.05 (close to Jupiter's) and 0.50 (greater than all the planets).

Our tests showed that if the stepsize was chosen so the local truncation error was less than machine precision, the root mean square (RMS) error in the energy and phase grew stochastically as $t^{1 / 2}$ and $t^{3 / 2}$ respectively.

The two-dimensional Kepler problem provides much insight about the Solar System over short intervals of time. On long intervals, inter-planetary forces are significant. Hence our test results from [8] are not applicable to long simulations of the Sun and several planets and it is necessary to directly test our method on these more realistic models.

We report on three such tests. The first test is a single 100 million year 
simulation of the Sun and the jovian planets (Jupiter, Saturn, Uranus and Neptune). This test demonstrates the stochastic growth of the error. The second test is sixteen simulations of the Sun and planets Venus through to Pluto over 322,000 years, and the third test sixteen simulations of the Sun and jovian planets over 800 million years. These two tests measure the RMS growth of the error. The initial conditions for all integrations were taken from the Jet Propulsion Laboratory (JPL) ephemeris.

The accuracy of simulations of the Solar System is typically measured using the error growth in the energy and other conserved quantities $[13,14$, 15, 17, e.g.]. However, resonances between planets such as the 3:2 resonance of Pluto and Neptune are important in the long term behaviour of the Solar System. If the phase error in a simulation is too large, these resonances will be destroyed, leading to erroneous results such as Pluto colliding with, or being scattered by, Neptune. Hence we use the phase error as well as the error in the energy to measure the accuracy of numerical solutions.

\section{Test 1}

We proved analytically in [8] for the two-body problem with eccentricities up to 0.5 , that the local truncation error for the order thirteen Störmer method was less than the unit round-off in IEEE double precision if the stepsize was less than one-thousandth of the period. The orbital eccentricities of the jovian planets are all less than 0.1 and the shortest orbital period is that of Jupiter, 4333 days. Hence, for a simulation of the Sun and jovian planets, our analytical result in [8] strongly suggests that if the stepsize is less than 4.333 days, the local truncation error will be less than unit round-off. We were conservative and used a stepsize of 4 days.

Figure 1 gives the relative error $e_{\mathrm{E}}$ in the energy. To provide a comparison, we added the curve for $\alpha t^{1 / 2}$, where $\alpha$ is chosen so $\alpha t^{1 / 2}$ interpolates the first 
data point for $e_{\mathrm{E}}$. The error $e_{\mathrm{E}}$ was defined as

$$
e_{\mathrm{E}}=\frac{e_{n}(t)-e_{n}(0)}{e_{n}(0)}
$$

where $e_{n}(t)$ is the energy at time $t$ calculated using the numerical solution. The error was calculated every 10,000 years, giving 10,000 data points.

If $e_{\mathrm{E}}$ was dominated by truncation error or systematic round-off error, it would grow approximately as $t$, and if it was dominated by random round-off error, approximately as $t^{1 / 2}$. Figure 1 shows $e_{\mathrm{E}}$ grows stochastically and lies below the curve $\alpha t^{1 / 2}$ most of the time. We used least squares to fit the power law $\gamma t^{\beta}$ to $e_{\mathrm{E}}$ and obtained $\beta=0.37$; this is further evidence that the error $e_{\mathrm{E}}$ is dominated by random round-off error. We repeated the calculations for the error in the total angular momentum and obtained $\beta=0.44$.

Figure 2 gives the phase error for Jupiter, Saturn and Uranus, together with the curve for $\alpha t^{3 / 2}$, where $\alpha$ is chosen so $\alpha t^{3 / 2}$ interpolates the first data point for Jupiter. The phase error for Neptune was omitted because it did not add materially to our conclusion and its inclusion would have crowded the figure. The phase error for the three planets was calculated using the difference between the numerical solution and a reference solution. The reference solution was found by an accurate integration in quadruple precision using a new variable-stepsize pair from the family of 10-12 explicit Runge-Kutta Nyström pairs derived by Dormand et al. [3]. The coefficients of this pair are available from the authors.

If the phase errors were dominated by truncation error or systematic round-off error, they would growth approximately as $t^{2}$, and if they were dominated by random round-off error, approximately as $t^{3 / 2}$. Figure 2 shows the phase errors grow slower than $t^{3 / 2}$ and the growth, particularly for Saturn, is stochastic. 


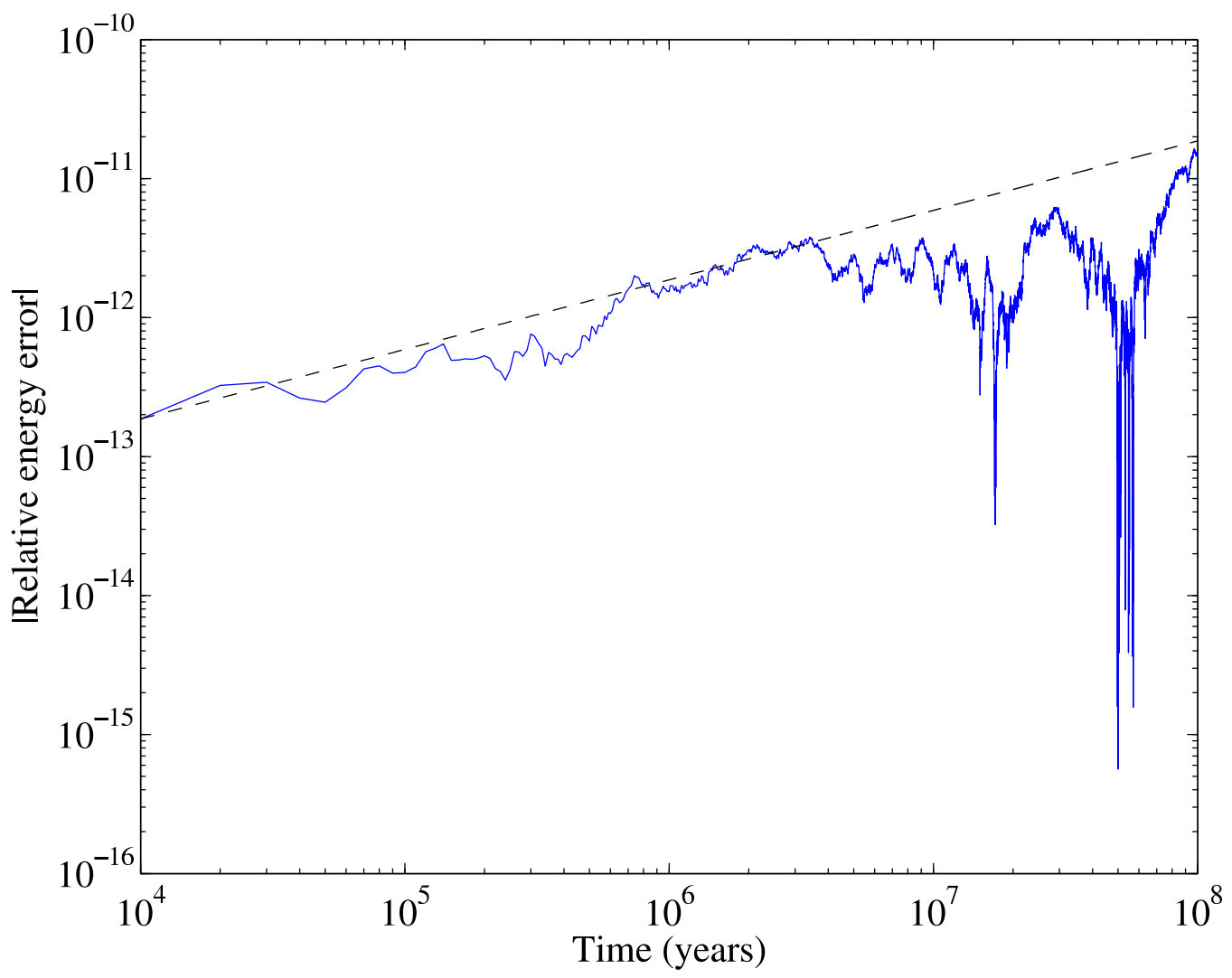

Figure 1: Solid line: the error in the energy. Dashed-line: $\alpha t^{1 / 2}$. 


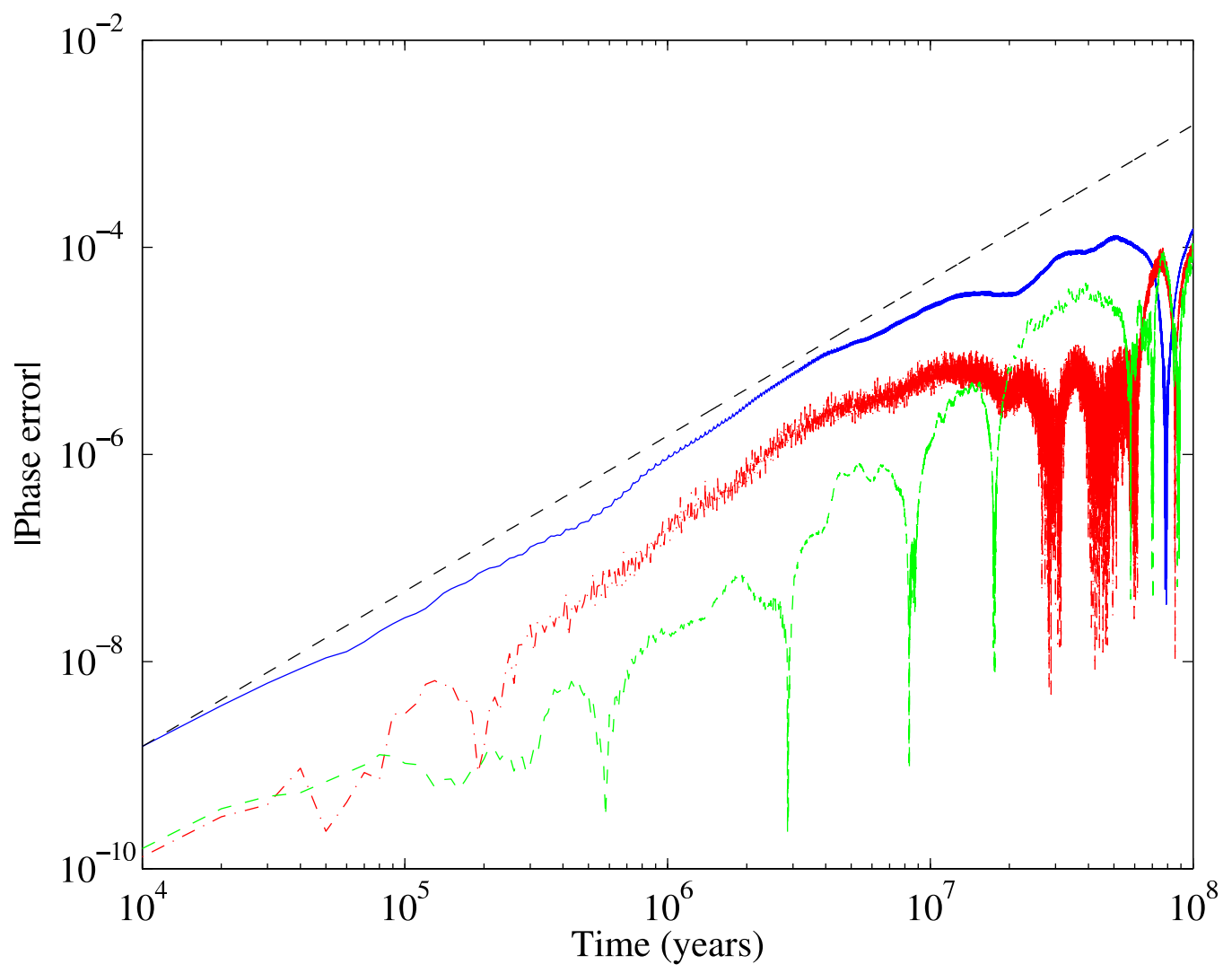

Figure 2: Upper dashed line, $\alpha t^{3 / 2}$; solid line, phase error for Jupiter; dashed-dotted line, phase error for Saturn; lower dashed line, phase error for Uranus. 


\section{Test 2}

For our second test, we performed sixteen integrations of the Sun and the planets Venus through to Pluto (Mercury's mass was added to that of the Sun). Venus's orbital period of 225 days is the smallest of the eight planets and we used a stepsize of 0.2 days. The initial conditions were taken from the JPL ephemeris at random multiples of ten years.

Quadruple precision on our computer is over twenty times slower than double precision. This meant it was impractical to find a reference in quadruple precision for each of the sixteen integrations. Instead, for each integration, we integrated for a time interval equivalent to $2^{i}$ Venusian orbits, where $i$ is an integer from 0 to 18 , and then integrated back to $t=0$. The difference between the numerical solution at $t=0$ and the initial conditions estimates the phase error. There is no systematic cancellation of the error on the backwards integration in our simulations because the error grows stochastically.

The RMS relative energy error for the planets is plotted in Figure 3. The error, consistent with our results for the two-dimensional Kepler problem, grows at a rate less than $t^{1 / 2}$ and after 524,288 orbits of Venus (approximately 322,000 years) we have a relative energy error of $2.4 \times 10^{-12}$. The quantitative difference between our observed error growth and $t^{1 / 2}$ is likely due to the finite number of integrations we performed; our previous paper showed this difference was to be expected.

The RMS phase errors for the terrestrial and jovian planets are plotted in Figures 4 and 5 respectively. The phase error for all eight planets including Pluto (not shown) grew at rates less than $t^{3 / 2}$. After $2^{18}$ orbits of Venus, the phase error for Jupiter was approximately $2.1 \times 10^{-7}$ radians. Extrapolated to one billion years, this yields an error of less than one degree. Kinoshita and Nakai [13] reported an error in Jupiter's longitude of 0.83 degrees over five million years; Applegate et al. [1] list their error in Jupiter's position as 100 degrees in 100 million years. 


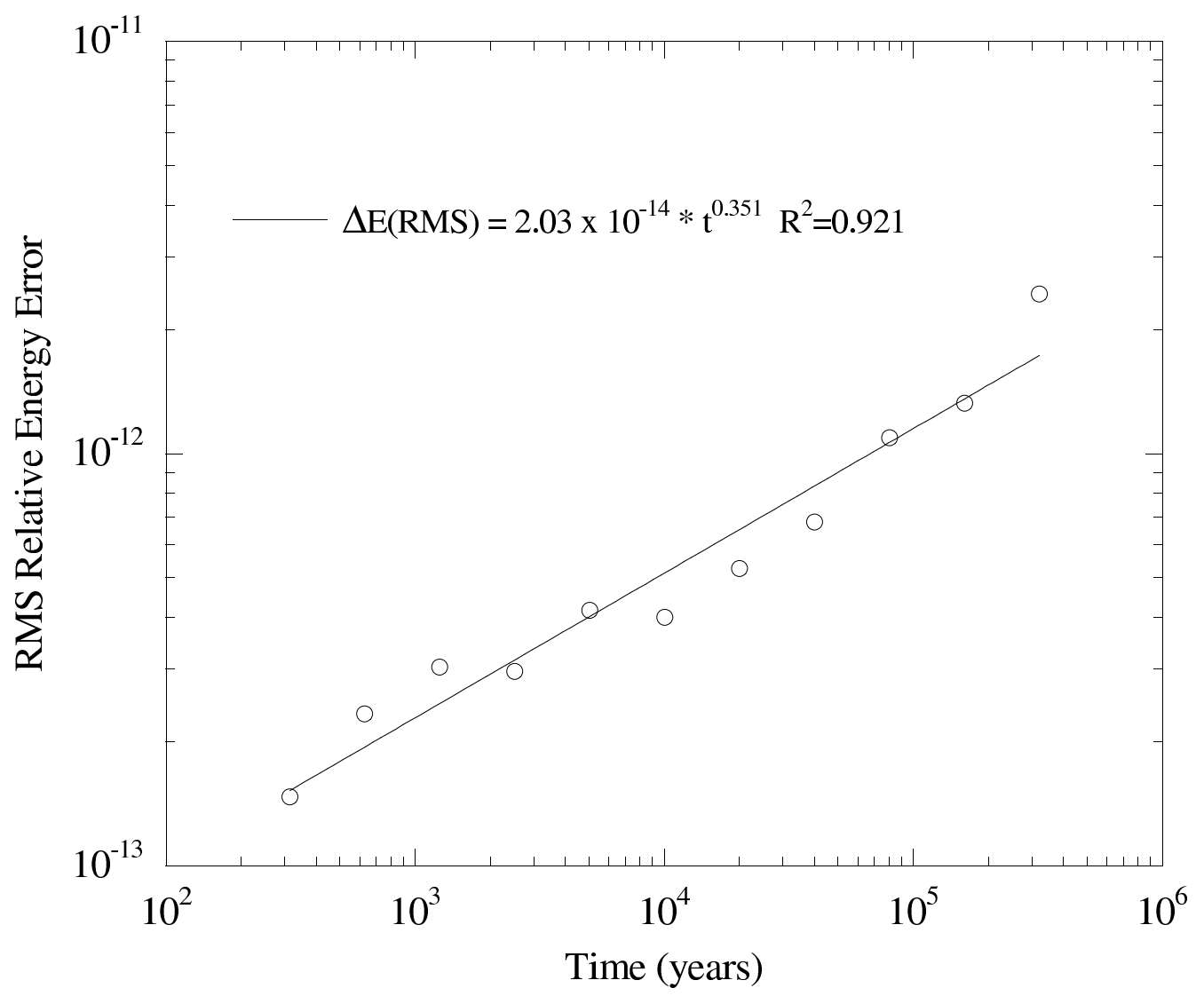

Figure 3: The RMS relative energy error for the entire Solar System. 


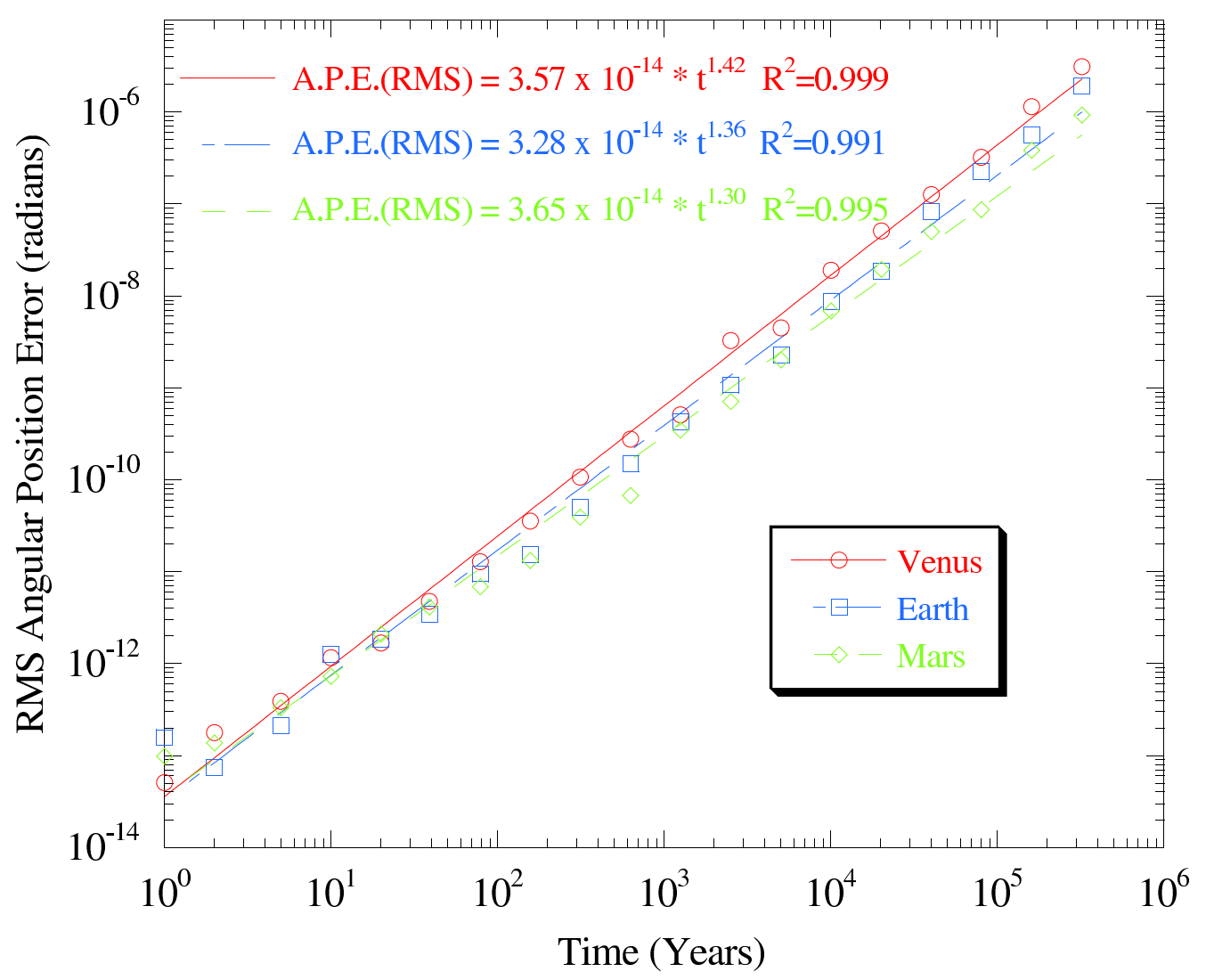

FiguRE 4: The RMS angular position error (phase error) for the terrestrial planets. 


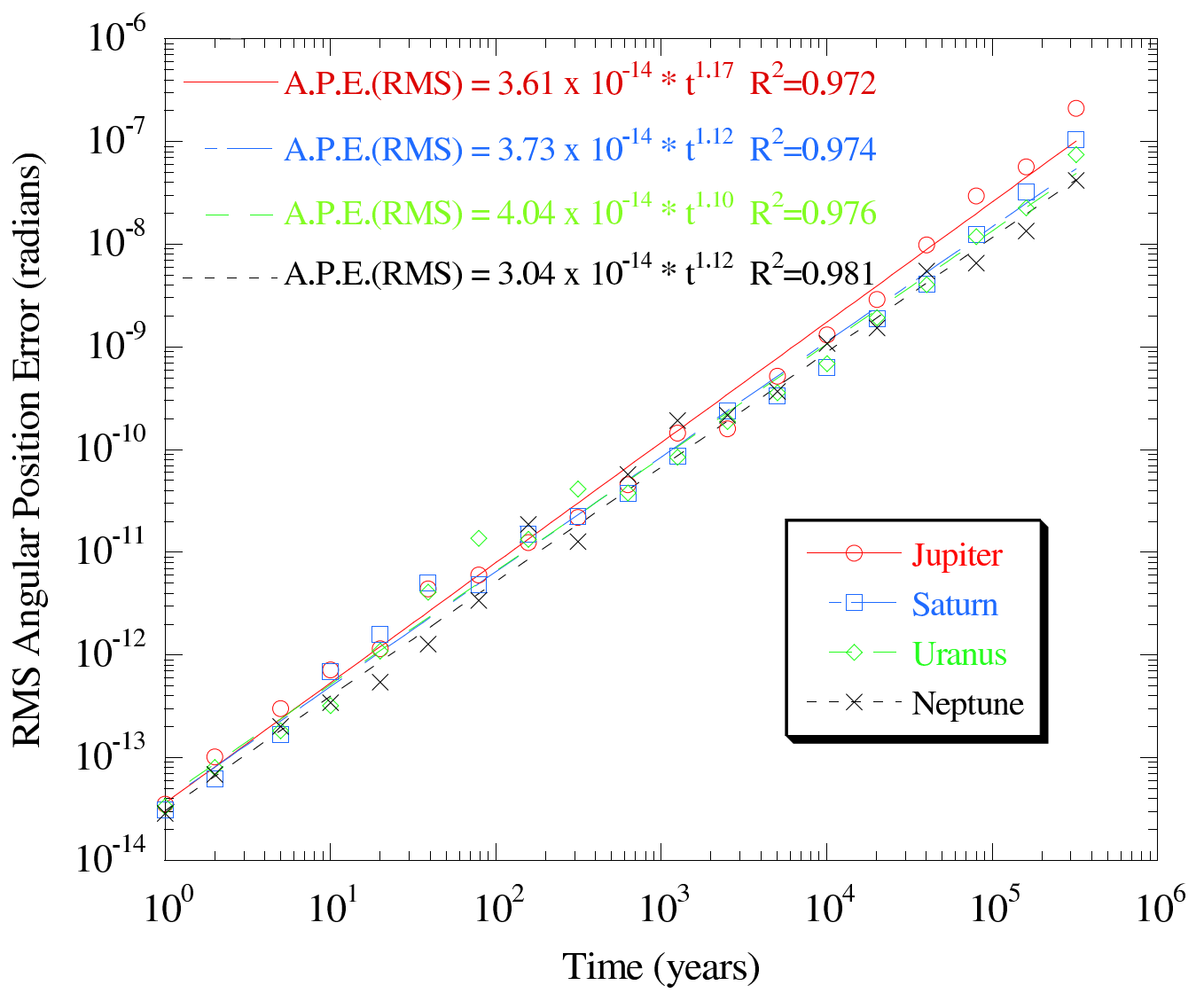

FiguRE 5: The RMS angular position error (phase error) for the jovian planets. 


\section{$4 \quad$ Test 3}

Our last test was sixteen integrations of the Sun and jovian planets, with the mass of the terrestrial planets added to that of the Sun and the initial conditions chosen in a way similar to that for Test 2 . We integrated the trajectories of the jovian planets for a time interval equivalent to $2^{i}$ Jupiter orbits, where $i$ is an integer between 0 and 25 . At the end of each integration, we used the positions and velocities of the Sun and planets as initial conditions to integrate backwards in time to $t=0$. The phase errors were then calculated as in Test 2 .

Figure 6 shows the RMS energy error for the entire system. We observe the relative energy error grows as $t^{0.48}$, very nearly $t^{1 / 2}$, indicating the absence of systematic error growth. Given that we are using a finite number of integrations, our results agree very well with theory.

Figure 7 shows the RMS phase errors for all jovian planets. The errors grow at rates less than $t^{3 / 2}$ and after $2^{26}$ Jupiter orbits $\left(2^{25}\right.$ orbits forward and $2^{25}$ orbits backwards, nearly 800 million years), the error for all planets is less than $1.9 \times 10^{-2}$ radians. Extrapolated, the phase error after $10^{9}$ years is less than $2^{\circ}$ for all planets, in good agreement with the extrapolated value of Test 2.

\section{Discussion}

We performed long simulations of the Sun and planets using an order thirteen Störmer method that had been implemented to minimise round-off error. The stepsize for each simulation was approximately one-thousand of the smallest orbital period. We found the error in the energy and phase grew approximately as $t^{1 / 2}$ and $t^{3 / 2}$ respectively. We thus achieved the lower bounds of Brouwer [2] and confirmed our earlier results [8] for the two-dimensional 


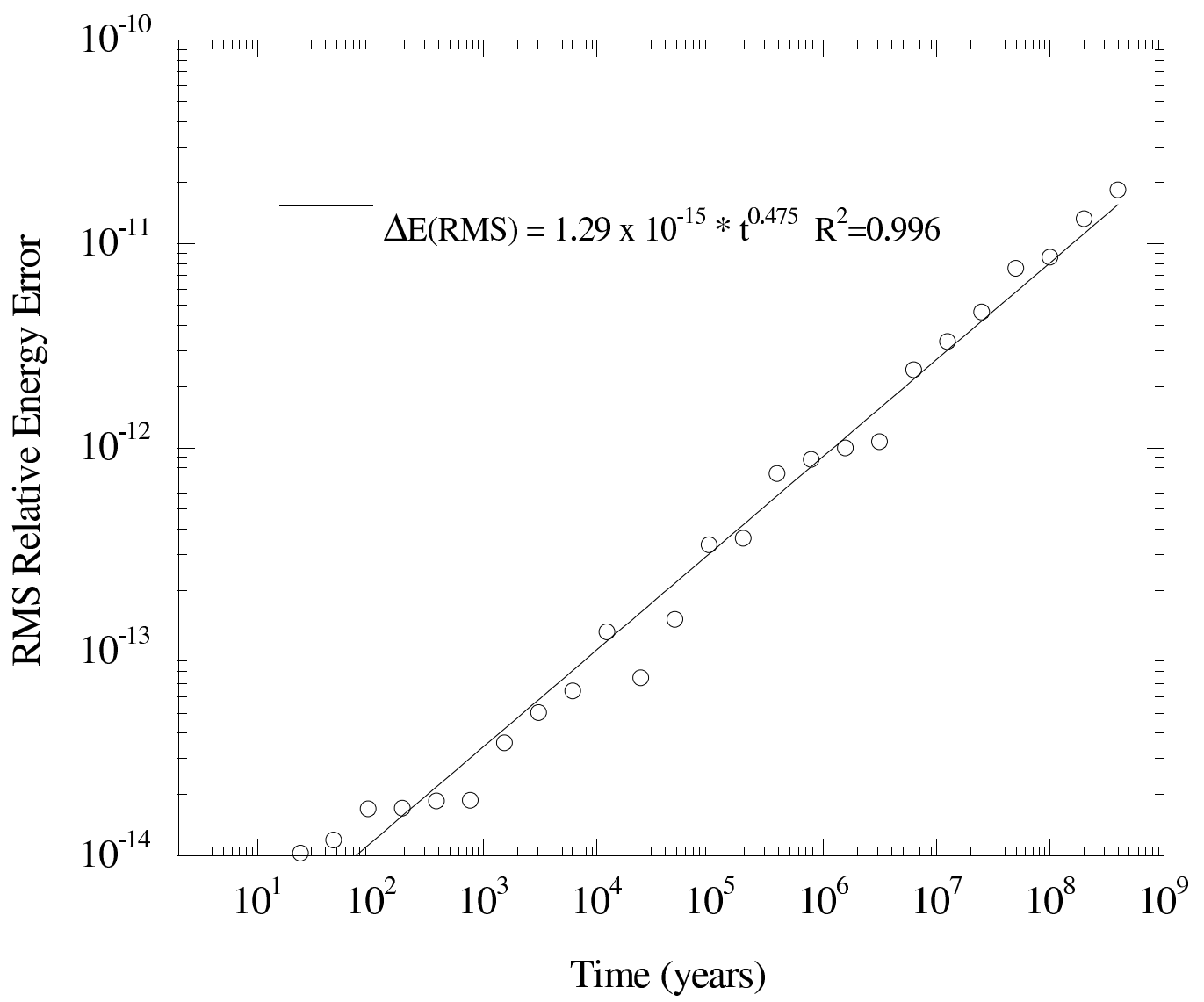

FiguRE 6: The RMS relative energy error for the outer Solar System. 


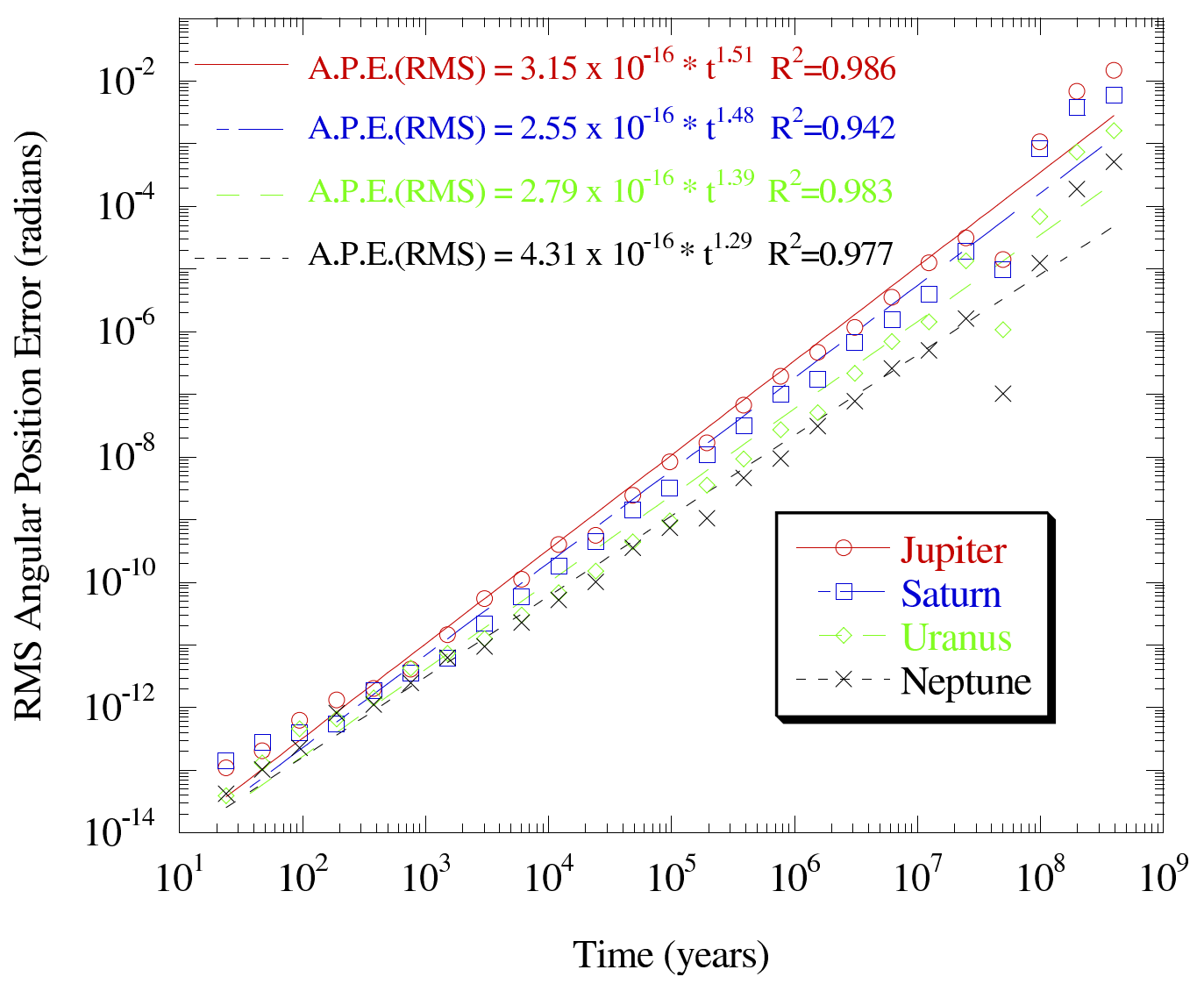

FiguRE 7: The RMS angular position error (phase error) for the outer Solar System. 
Kepler problem.

The aim of the present work and that in [8] was to implement high order Störmer methods in a way that simulations of the planets and planetesimals were as accurate as possible for the computer being used. We showed this was possible if the stepsize was chosen so the local truncation error of the Störmer method was less the machine precision. This necessitates a small stepsize but, as the work in [6] and [7] shows, the stepsize is large enough that simulations of a large number of planetesimals over long intervals are possible on modern workstations.

As well as demonstrating Brouwer's Law is achievable for realistic simulations, our results for our second and third tests point to another implication. Previous studies such as in [16] reported the outer Solar System is chaotic. Chaotic systems are characterized by an exponential divergence of nearby trajectories, and have both stable and unstable manifolds. In particular, suppose we select different initial conditions and compute the orbit for some length of time, then reverse the direction of the integration in order to identify how close we are upon return to the initial conditions. During the course of the computation, we expect that accumulated integration error will cause the computed solution to depart from the exact solution associated with the initial condition. This means the integrated solution represents a nearby trajectory to the true solution. If a set of initial conditions is representative of a chaotic system - that is, an unstable manifold is present - nearby orbits would show exponential divergence from the exact solutions for integrations both forward and backward, and would return to the starting point with an error that has grown exponentially in time. The rate that error grows in time would be characterized by the same Lyapunov time that characterizes the chaotic character of the system. If the system is not chaotic - that is, no unstable manifold exists - the divergence of the final computed solution from the initial conditions after the return integration will not depend upon the elapsed time exponentially, but will vary as a power-law in elapsed time (see also [17]). If an unstable manifold was present, only a set of measure 
zero of initial conditions (in the presence of computational error) would not show exponential divergence. None of our randomly selected initial conditions showed chaotic behaviour.

In addition, the errors in the energy and angular momentum for the quadruple precision reference solution of Test 1 both grew as $t^{1.09}$, good agreement with the expected linear growth for a non-chaotic system. We checked the reference solution further by calculating a more accurate reference solution and using this to estimate the global error in the less accurate reference solution. The $L_{2}$ norm of the global error for the five bodies grew as the power law $t^{2.07}$, good agreement with the expected quadratic growth for a non-chaotic system.

Our conclusion about the stability of the outer Solar System agrees with that of Ito and Tanikawa [12]. They performed two simulations of the Jovian planets and Pluto that spanned $5 \times 10^{10}$ years, approximately 10 times the age of the Solar System. One simulation was forward in time, the other backwards in time. The initial conditions for their simulations were taken from the same JPL ephemeris we used. Ito and Tanikawa concluded the outer planetary system was stable for $5 \times 10^{10}$ years.

Acknowledgment: We thank the three referees for their careful reading of the submitted paper and their suggestions about improving. We are particularly grateful to Ferenc Varadi for much discussion. This research was carried out in part at the Jet Propulsion Laboratory, California Institute of Technology, and was supported in part by NASA NAGW to UCLA.

\section{References}

[1] J. H. Applegate, M. R. Douglas, Y. Gürsel, G. J. Sussman and J. Wisdom, The outer Solar System for 200 million years, Astron. J. 92 
(1986), 176-194. C1093

[2] D. Brouwer, On the accumulation of errors in numerical integration, Astron. J. 46 (1937), 149-153. C1088, C1097

[3] J. R. Dormand, M. E. A. El-Mikkawy, P. J. Prince, High-order embedded Runge-Kutta-Nystrom formulae, IMA J. Numer. Anal. 7, (1987), 423-430. C1090

[4] M. Duncan, T. Quinn and S. Tremaine, The long-term evolution of orbits in the Solar System: a mapping approach, Icarus 82 (1989), 402-418. http://dx.doi.org/10.1016/0019-1035(89)90047-X C1088

[5] K. R. Grazier, The stability of planetesimal niches in the outer Solar System: a numerical investigation, $\mathrm{PhD}$. dissertation, Department of Mathematics, University of California, Los Angeles, 1997. C1088

[6] K. R. Grazier, W. I. Newman, W. M. Kaula and J. M. Hyman, Dynamical evolution of planetesimals in the outer Solar System. I. The Jupiter/Saturn Zone. Icarus 140 (1999), 341-352. http://dx.doi.org/10.1016/0019-1035(89)90047-X C1088, C1100

[7] K. R. Grazier, W. I. Newman, F. Varadi, W. M. Kaula and J. M. Hyman, Dynamical evolution of planetesimals in the outer Solar System. II. The Saturn/Uranus and Uranus/Neptune Zones. Icarus 140 (1999), 353-368.

http://dx.doi.org/10.1016/0019-1035(89)90047-X C1088, C1100

[8] K. R. Grazier, W. I. Newman, J. M. Hyman, P. W. Sharp, and D. G. Goldstein, Achieving Brouwer's Law with high-order Störmer multistep methods, ANZIAM J., 46(E) (2005) C786-C804. http://anziamj.austms.org.au/V46/CTAC2004/Graz C1088, C1089, C1097, C1100 
[9] E. Hairer, S. P. Nørsett and G. Wanner, Solving ordinary differential equations I, 2 ed, Springr-Verlag, 1993. URL C1088

[10] P. Henrici, Discrete variable methods in ordinary differential equations, John Wiley \& Son, New York, 1962. C1088

[11] M. J. Holman, A possible long-lived belt of objects between Uranus and Neptune, Nature 387 (1997) 785-787. http://dx.doi.org/10.1038/42890 C1088

[12] T. Ito and K. Tanikawa, Long-term integrations and stability of planetary orbits in our Solar System, Mon. Not. R. Astron. Soc. 336 (2002), 483-500.

http://dx.doi.org/10.1046/j.1365-8711.2002.05765.x C1101

[13] H. Kinoshita and H. Nakai, Motions of the Perihelions of Neptune and Pluto Celest. Mech. 34 (1984), 203-217. C1089, C1093

[14] A. Milani and A. Nobili, Integration error over very long time spans, Celest. Mech. 43 (1988) 1-34. C1089

[15] G. Quinlan and S. Tremaine, Symmetric multistep methods for the numerical integration of planetary orbits, Astron. J. 100 (1990), 1694-1700. C1089

[16] G. Sussman and J. Wisdom, Chaotic evolution of the Solar System, Science, 257 (1992), 56-62. C1100

[17] H. Yoshida, Non-existence of the modified first integral by symplectic integration methods II: Kepler problem, Celest. Mech. Dyn. Astron. $\mathbf{8 3}$ (2002), 355-364. http://dx.doi.org/10.1023/A:1020158625544 C1089, C1100 